\title{
INTELLIGENT ADAPTRONICS FOR MEMS AND NEMS
}

\author{
GHEORGHE, G.; VOICU, A.; ILIE, I.; \\ GornoAva, V. \& CONSTANTIN, A.
}

Abstract: The scientific paper "Intelligent Adaptronics for MEMS and NEMS", treats the integrating concepts and technical and technological fusions of Mechatronics, Integronics and Adaptronics, with theoretical and practical applications in the construction of MEMS and NEMS implemented in industry, economy and society The scientific paper "Intelligent Adaptronics for MEMS and NEMS " treats integrating concepts and technical and technological mergers in theoretical and practical applications for MEMS and NEMS building, with a strong knowledge base that spans modelling, simulation, fabrication and links between design manufacture and performance.

Key words: adaptronics, mechatronics, integronics, MEMS, NEMS
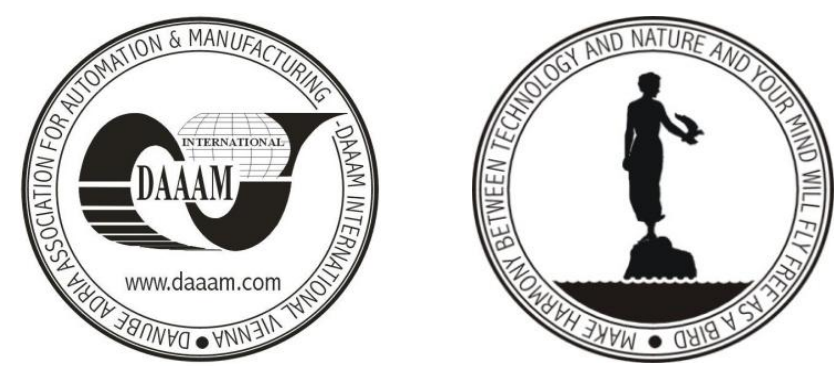

Authors' data: Univ. Prof. Ph'D. Eng. Gheorghe, G[heorghe]*; Eng. Voicu, A[drian $]^{* *}$, Eng. Ilie, I[ulian]**, Eng. Gornoava, V[alentin]**, Eng. Constantin, A[nghel]**, *National Institute of Research and Development in Mechatronics and Measurement Technique, Pantelimon Road, 6-8, 021631, Bucharest, **Valahia University of Targoviste, B-dul Carol I,2. 130024,Academy of Technical Sciences of Romania, B-dul Dacia 26, Bucharest, Romania, geocefin@yahoo.com, adrian.ktlin@yahoo.com, iuly.ily@yahoo.com, valentin_gornoava@yahoo.com, anghel.constantin@incdmtm.ro

This Publication has to be referred as: Gheorghe, G[heorghe]; Voicu, A[drian Catalin]; Ilie, I[ulian]; Gornoava, V[alentin] \& Anghel, C[onstantin] (2014). Intelligent Adaptronics for Mems and Nems, Chapter 01 in DAAAM International Scientific Book 2014, pp.001-018, B. Katalinic (Ed.), Published by DAAAM International, ISBN 978-3-901509-98-8, ISSN 1726-9687, Vienna, Austria DOI:10.2507/daaam.scibook.2014.01 
Gheorghe, G.; Voicu, A. C.; Ilie, I.; Gornoava, V. \& Anghel, C.: Intelligent Adaptr...

\section{Introduction}

MEMS is a relatively new area in engineering. Experimental and analytical systematic research on MEMS started around 1970s as an investigative interest of Si foundry engineering to the quite peculiar properties of mono-crystalline Si. MEMS gained significant momentum when useful devices became commercially available. The research on MEMS (Gheorghe \& Badita, 2013) gained more significance through the subdivision of interest which has created a solid base of knowledge that is spread over modelling, simulation, manufacturing and the liaison among design, fabrication and performances. Each research area has brought light over either fabrication methods (extension of MEMS from $\mathrm{Si}$ to metal or/and polymer), modelling principles, mathematical description of multi-physics phenomena, test methodology and characterization, etc. The new designs target specific applications that originally were strongly bonded to mass production systems. The significant advancement made by the fabrication technologies including the multi-user concept made possible to conceive MEMS for lower production applications. The unusual physics of microstructures played a major role mainly in the development of new MEMS used for detection and sensing.

The modelling of MEMS comes with some significant challenges. The unusual influences of specific field forces or the significantly relative large defections encountered by the mechanical microstructures make the researchers assume that the macro model constituent equations may not always accurately describe some of the phenomena. Most of the present works in modelling assume that microstructures behave as large scaled structures of the same type while scaled down. It is apparent from here that using nonlinear formulations may enhance the accuracy of the models. The non-linear mechanics is a well-established area in which the contributions are usually brought by theoreticians. Practical investigations on MEMS have been so far focused by experimentalists.

The objective of this research is to investigate how nonlinearities affect both static and dynamic performance of the microstructures in comparison with the linearity assumptions. The specific objective of the research is to prove that the assumption of non-linearity significantly improves the models accuracy. The results of the non-linear models will be compared with the experimental data and weigh against the linear based modelling of few popular microstructures including cantilever beams, cantilever bridges and micro-plates will be evaluated and accurate nonlinear models will be proposed.

\section{Nonlinearity effects on static and dynamics performance of MEMS}

\subsection{Nonlinearity}

First, one has to define the meaning of nonlinearity in mechanics analysis. For a physical phenomenon in which a single input variable is targeted and a single input is sought, a generic mathematical model can be written. This mathematical model is usually an algebraic equation like: 


$$
x_{k+1}=f\left(x_{k}\right)
$$

which is called discrete-time system (Gheorghe et al., 2011). For continuous - time systems the mathematical model is a differential equation in the form:

$$
\dot{x}=f(x, t)
$$

that is called non-autonomous system. In an autonomous system the differential is the form:

$$
\dot{x}=f(x, M)
$$

where in all of above equations, $f$ is a map from manifold $M$ to $N, M \subset R^{n}$ and $N \subset R^{n}$. If in a continuum mechanics the differential equations describing the governing phenomena are linear, the problem will be called linear. Otherwise they are called nonlinear. There are two sources of nonlinearity in mechanical engineering modelling. (a) Geometric (b) Material. Geometrical nonlinearity is due to the nonlinear stress-strain relation or to large deformation while material nonlinearities are assigned to the nonlinear behavior of material properties.

However, in most cases, simplifying problem to linear differential equation help to solve the model much easier than nonlinear differential equations and in most cases the results are acceptable within certain range. However, there are some cases that simplifying of differential equations to linear form creates incorrect answers. These cases are difficult to point before the validation against experimental results is performed.

\subsection{Geometric nonlinearity}

Geometric nonlinearities (Belendez, 2002) are mainly due to the fact that the deformation gradient is very large in comparing to the size of the body or otherwise said, the stress and the strain do not keep a linear relation like Hooks law. So it is necessary to define new strain and stress relationship. To define the strain in material coordinate, it is necessary first to define stretch ratio:

$$
\lambda_{a_{0}}\left(X_{P}, t\right) F\left(X_{P}, t\right) a_{0}
$$

Where:

$F$ is the deformation gradient,

$X_{P}$ is the position vector,

$a_{0}$ is the unit vector,

Where $\mathrm{F}$ is defined as:

$$
F=\left[\frac{\partial X_{P}}{\partial x}\right]
$$


Gheorghe, G.; Voicu, A. C.; Ilie, I.; Gornoava, V. \& Anghel, C.: Intelligent Adaptr...

Green deformation tensor (Right Cauchy-Green tensor) is defined as:

$$
C=F^{T} F
$$

And Green-Lagrange strain tensor is defined as:

$$
E=\frac{1}{2}\left(F^{T} T-I\right)
$$

One dimension Green-Lagrange strain is:

$$
\varepsilon_{G}=\frac{l^{2}-L^{2}}{2 L^{2}}
$$

Where $\mathrm{L}$ is initial length and 1 is final length. Strain in spatial coordinate, defined as:

$$
b=F F^{T}
$$

$\mathrm{b}$ is left Cauchy-Green tensor. Euler-Almansi strain tensor is defined as:

$$
e=\frac{1}{2}\left(I-F^{-T} F^{-1}\right)
$$

One dimension Euler-Almansi strain is:

$$
\varepsilon_{A}=\frac{l^{2}-L^{2}}{2 l^{2}}
$$

At this point, stress tensor should be defined for nonlinear stress analysis.

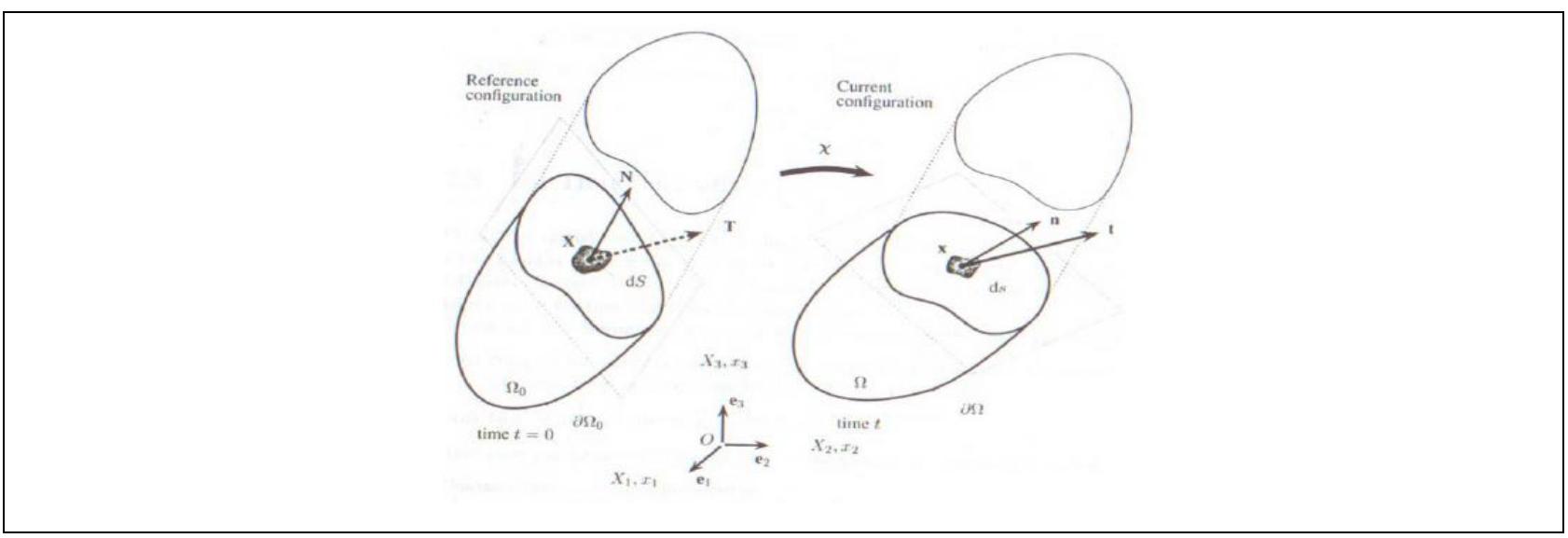

Fig. 1. Traction vectors acting on a surface element

As it is clear from the above figure, for an element one can write:

$$
d f=t_{C} d s=T_{P} d s
$$




$$
t_{C}=t_{C}(x, \tau, n) T_{P}=T_{P}(X, \tau, N)
$$

where $t_{C}$ is Cauchy (or true) traction vector (force measured per unite surface area defined in the current configuration) exerted on $d s$ with normal $n . T_{P}$ is the first PiolaKirchhoff (or normal) traction vector. There exist unique second-order tensor fields $\sigma$ and $P$ so that:

$$
\begin{gathered}
t_{C}(x, t, n)=\sigma(x, t) n \\
T_{P}(x, t, N)=P\left(X_{P}, t\right) N
\end{gathered}
$$

Where $\sigma$ is called Cauchy stress tensor. These equations show that, if traction vectors depend on unites normal then they must be linear in normal.

\subsection{Material nonlinearity}

Material nonlinearity is due to nonlinear constitutive behavior of material of the system. Among the nonlinearity of materials, hyperelasticity of materials is of at most importance in mechanical micro-structures. Many microstructures can be simulated by hyperelastic behavior of materials. These kinds of materials have large elastic strain which is recoverable (Smith et al., 1995). It is shown that the stress response of hyperelastic material is derived from the given strain-energy function W. Numerous forms of strain-energy functions have been introduced. For using these forms, we have to define strain potential energy. $\mathrm{W}$ is a scalar function of strain or deformation tensors which one can write:

$$
S_{i j}=\frac{\partial W}{\partial E_{i j}}=2 \frac{\partial W}{\partial C_{i j}}
$$

Where:

$S_{i j}$ are components of second Piola-Kirchhoff stress tensor

$W$ is strain energy function per unit undeformed volume

$E_{i j}$ are components of the Green-Lagrange strain tensor

$C_{i j}$ are components of right Cauchy-Green tensor

Under the assumption that material response is isotropic, it is convenient to express the strain energy function in terms of strain invariants:

$$
W=W\left(I_{1}, I_{2}, I_{3}\right)=W\left(I_{1}, I_{2}, \lambda\right)=W\left(\lambda_{1}, \lambda_{2}, \lambda_{3}\right)
$$

Where:

$$
\begin{aligned}
& I_{1}=\lambda_{1}^{2}+\lambda_{2}^{2}+\lambda_{3}^{2} \\
& I_{2}=\lambda_{1}^{2} \lambda_{2}^{2}+\lambda_{2}^{2} \lambda_{3}^{2}+\lambda_{3}^{2} \lambda_{1}^{2} \\
& I_{3}=\lambda_{1}^{2} \lambda_{2}^{2} \lambda_{3}^{2}=J^{2}
\end{aligned}
$$


Gheorghe, G.; Voicu, A. C.; Ilie, I.; Gornoava, V. \& Anghel, C.: Intelligent Adaptr... And $I_{1}, I_{2}, I_{3}$ are invariants of $C$, also $\lambda_{1}^{2}, \lambda_{2}^{2}, \lambda_{3}^{2}$ are eigen-values of $C$. By defining the volume-preserving part of, $F$ :

where:

$$
\bar{F}=J^{-\frac{1}{3}} F
$$

$$
J=\operatorname{det}(F)
$$

it can be shown that:

$$
\begin{gathered}
\bar{\lambda}_{p}=J^{-\frac{1}{3}} \lambda_{p} \quad p=1,2,3 \\
\bar{I}_{p}=J^{-\frac{2}{3}} I_{p}
\end{gathered}
$$

Therefore, the strain potential energy is:

$$
W=W\left(\bar{I}_{1}, \bar{I}_{2}, J\right)=W\left(\bar{\lambda}_{1}, \bar{\lambda}_{2}, \bar{\lambda}_{3}, J\right)
$$

Here, we introduce some models that are defined for strain energy potential applications. These models proved to be quite appropriate to model strong non-linear strain-stress relationships.

\section{A) - Neo-Hookean model:}

Where:

$$
W=\frac{\mu}{2}\left(\bar{I}_{1}-3\right)+\frac{1}{d}(J-1)^{2}
$$

$\mu=$ initial shear modulus of materials

$\mathrm{d}=$ material incompressibility

B) - Mooney-Rivlin:

$$
\begin{aligned}
& W=C_{10}\left(\bar{I}_{1}-3\right)+C_{01}\left(\bar{I}_{2}-3\right)+C_{20}\left(\bar{I}_{1}-3\right)^{2}+ \\
& C_{11}\left(\bar{I}_{1}-3\right)\left(\bar{I}_{2}-3\right)+C_{02}\left(\bar{I}_{2}-3\right)^{2}+C_{30}\left(\bar{I}_{2}-3\right)^{3}+ \\
& C_{21}\left(\bar{I}_{1}-3\right)^{2}\left(\bar{I}_{2}-3\right)+C_{12}\left(\bar{I}_{1}-3\right)\left(\bar{I}_{2}-3\right)^{2}+C_{03}\left(\bar{I}_{2}-3\right)^{3}+\frac{1}{d}(J-1)^{2}
\end{aligned}
$$

Where:

$$
C_{10}, C_{01}, C_{20}, C_{11}, C_{02}, C_{30}, C_{21}, C_{12}, C_{03}, d=\text { material constants }
$$

C) - Ogden potential

$$
W=\sum_{i=1}^{N} \frac{\mu_{i}}{\alpha_{i}}\left(\bar{\lambda}_{1}^{\alpha_{i}}+\bar{\lambda}_{2}^{\alpha_{i}}+\bar{\lambda}_{3}^{\alpha_{i}}-3\right)+\sum_{k=1}^{N} \frac{1}{d_{k}}(J-1)^{2 k}
$$

Where:

$\mathrm{N}, \mu_{i}, \alpha_{i}, d_{k}=$ material constant

\subsection{Cantilever beams nonlinear deflection}

Most of mathematical close forms formulations on nonlinear deflections of cantilever beams are just for point force on the tip. The only different aspect is the 
approach of the formulation of the solving algorithm. For a moment on the beam, it is possible to reduce one degree of ODE by Lie symmetry. The result is:

$$
\frac{2 E I\left(y-x y^{\prime}\right)}{\sqrt{1+y^{\prime 2}}}-M\left(x^{2}+y^{2}\right)-(E I)^{2}=\text { const }
$$

There has been significant research carried out on micro-cantilever beams. Most of these studies are on the influence of the electrostatic forces and the adhesion or stiction of beams. All works in which the models were formulated in non-linear equations were solved by numerical methods and compared with experimental results. Many investigations in micro-level modelling are looking at the dynamic properties and behavior of micro-cantilevers beams. Natural frequency as well as the quality factor are used for controlling, for example, voltage, and switches, in acoustic wave resonators. Bimorph micro-cantilevers are more sensitive than anamorphic beams. In some works, researches showed that sensitivity increases in bimorph materials significantly. Bimorph micro-cantilever beams are mostly used in thermal sensing and for this purpose the tip deflection of the bimorph cantilever beams, large deflections, stress analysis and experimental validations were carried out. Bimorph micro-cantilever beams are also used as actuators. The mathematical model, of the deflection is studied. Thermal actuators are studied widely, theoretically, experimental applications and for their dynamic behavior.

Nonlinear dynamical behavior of micro-plates has been studied in Virginia Polytechnic Institute recently. Mostly the work is focusing on nonlinear response of micro-plates. Other mathematical methods are used in micro-plates such as BEM where the method is used just for electrical field formulation and then tractions on the plate surface.

\section{Microelectromechanic and nanoelectromechanic microsystems}

MEMS \& NEMS represents a relatively new concept, based on the structure of already integrated systems which include parts of precision mechanics, electric circuits, controllers and software programs.

The first vision about MEMS \& NEMS was made by Dr. Richard Feynman, through the problem of micro and nano objects control and manipulation. Through the implementation of micro and nanotechnologies, material, equipment and system production and manufacturing changed a lot through:

- Foreseen properties concerning nano components and micro materials (e.g.: light weight, high durability, thermal stability, small volume, small dimensions, high energetic power, torsion, power, charge and densities, specific thermal conductivity, etc.);

- The improvement of accuracy, very high precision, high durability and reliability;

- Higher degree of efficiency, capacity, flexibility, integrity;

- Higher degree of security;

- Competitive environment; 
Gheorghe, G.; Voicu, A. C.; Ilie, I.; Gornoava, V. \& Anghel, C.: Intelligent Adaptr...

Through MEMS \& NEMS development, a great number of applications could be reported, from which many were successful on the market and many other are still under investigation.

In perspective, the actual market shows an intensive MEMS \& NEMS growth and opens a large number of technical, technologic and innovative applications.

In MEMS \& NEMS design, one comes across with confrontational situations with respect to complex systems which imply physics, chemistry and phenomena which are unknown in the present or which are known but haven't been investigated in depth.

The foresight concerning MEMS \& NEMS' technological progress in the upcoming period of time has been directed not only towards their miniaturization but also towards advanced micro nanotechnologies application.

In the future MEMS \& NEMS vision, arise different problems to be solved, such as:

- Problems for which fundamental theories are developed, but the complexity of the solutions is behind actual or near future technologies, as it can be exemplified by means of molecular dynamics which cannot be applied even for nano scale operations;

- Problems for which fundamental theories are not completely-developed to justify direct simulations, but can be developed with the help of advanced number methods;

- Problems for advanced designed and stimulation methods will create important evolutions with a major impact.

The MEMS \& NEMS functional system has at its core various objectives, criteria, decisions and analyses which can be fulfilled through sensors, actuators, command, amplification and result. MEMS \& NEMS applications can be found in the complexity of process phenomena and component elements, with:

- Micro-nano-controllers and micro-nano-computers;

- Sensors, micro-nano-sensors and transducers, micro-nano-transducers;

- Vibrations, temperatures, pressures, speeds, accelerations, noises, radiations, etc. MEMS \& NEMS are constructive and parametric solutions, at micro and nano scale in order to be:

- Lighter;

- Cheaper;

- Faster;

- More efficient;

- With higher accuracy;

- Fitter.

MEMS represent:

- The emerging theory which improves miniature systems growth - microsystems which imply micromechanical and electric parts and orders on the same silicon chip; 
- The branch of IC technology and miniaturizing mechanics;

- The opening of new ways of understanding the phenomenon initiated at micro scale;

- Subjects related to miniaturization, such as electrostatic powers, high natural frequencies, etc.

In MEMS there are also novelties:

- The evaluation of microstructures' dynamic/ static behaviour;

- Microstructures' design; Microstructures' design depends on:

- The technology the systems were implemented with;

- Design configuration, as a challenge;

- Manufacturing achieved in adequate industrial processes;

- Post-processing.

For MEMS \& NEMS, the world in which they work is "the microscopic world", or "the world at micro and nano scale";

Therefore, in the brief history of MEMS \& NEMS, in 1959, Jack Kilby invented the monolithic integrated circuit, and the design was improved by Robert Noyce, in order to produce planar technology.

Further on, "the copy of the first transistor" is shown through the fabrication process called "photolithography":

- Engraving model and process;

- Photosensitive (photoelectric resistance) covering;

- Photoelectric resistance exposure through mask;

- Selective resistance;

- Substrate engraving;

- Photoelectric resistance band.

Generally, electric circuits are created "in planar processes", that is created and constructed at surface, by applying Si technology.

In order to create "micro-nano-products in three dimensions", "the LIGA technology" can be applied - micro-stereo-litogrphy, when MEMS and NEMS are obtained through micro-stereo-litography.

In order to identify their applications, MEMS \& NEMS' advantages are:

- Extremely good performances (low noise, high reliability, high natural frequency);

- Low energy consumption;

- Low mass/ inertia;

- Integration capacity;

- Small dimensions;

- Multifunctional;

Generally, the MEMS \& NEMS construction can be achieved through two approaches, volumetric processing and surface processing. 
Gheorghe, G.; Voicu, A. C.; Ilie, I.; Gornoava, V. \& Anghel, C.: Intelligent Adaptr...

For the approach of the things mentioned above, when designing MEMS \& NEMS a certain technology is aimed, the design concept is created, bearing in mind the technology, analyses for a structural solution are conducted and "the master mask" for the industrial manufacturing process is designed.

The MEMS \& NEMS manufacturing/ construction process can be developed as fallowing:

- The manufacturing submission is made - similar prototype;

- The testing matrix design is created;

- The post-processing of the active structure release is conducted;

- The packing testing is conducted - different than the one for service;

- Conclusions are drawn and it is decided whether a new design is needed.

MEMS \& NEMS reaches towards the fields which need these micro-nano-systems, such as:

- Oil and gas field - for intelligent drilling or for physical and chemical variables measuring;

- Renewable energy field - for steam/ heat sources monitoring;

- Aeronautics domain - for hot locations: motors, interrupted systems, etc.;

- Medical and biomedical field;

- Measuring, testing field;

- Communications field;

- Etc.

\subsection{MEMS Applications}

The science of materials, bio-technology, electronics and mechanical detection and stimulation will benefit from developments in micro-nano-robotics.

Research in bio-robotics includes the autonomous manipulation of cells or single molecules, characterization of mechanical properties of bio-membranes using micro-robotic systems with integrated vision and force detection modules. The scope of research is to obtain a fundamental understanding of the unicellular biological systems and ensuring mechanical models for the deformable trajectory of cells during bio-manipulation and study of injured cells. The manipulation of robotic at micronano-scale is a promising micro-nano-technology for the design, characterization and assembly of micro-nano groups in MEMS and NEMS. Combined with recently developed nano-fabrication processes, a hybrid mode is designed to build MEMS and NEMS and other micro-nano-robotic devices made of individual carbon nano-tubes and Ge / Si nano-coils.

\subsubsection{Bio-manipulation for autonomous robotic injection of DNA pronuclei}

To improve the low success rate of manual operation and to remove contaminants, an autonomous robotic system (shown in Fig. 2.) was developed to deposit DNA in one of the two nuclei of an embryo without inducing cell damage. Laboratory experimental results show that the success rate for the autonomous injection of DNA in the pronucleus is considerably improved in respect to 
conventional injection methods. The micro-robotic autonomous system characterizes a hybrid controller combining a visual system and precision position control, and pattern recognition to detect nuclei and a precise auto focusing scheme. Fig. 3. illustrates the injection process.

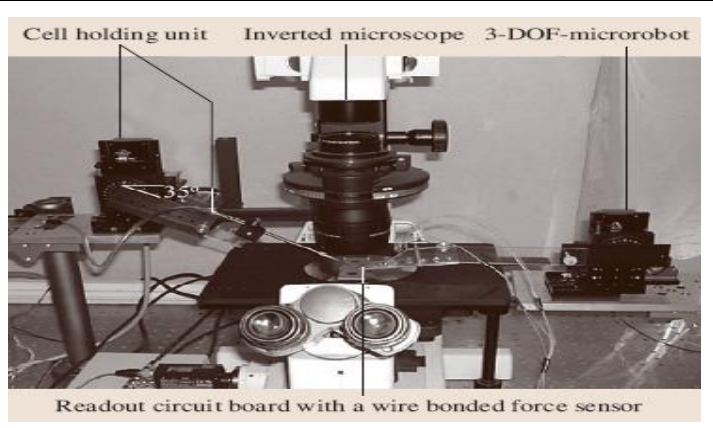

Fig. 2. Miro-nanorobotic bio-manipulation system with image and reaction force

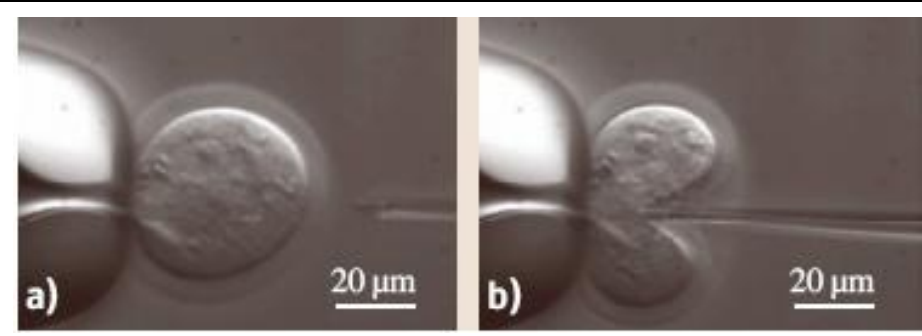

Fig. 3. Cell injection process. (A, b) Injection of an oocyte cell

To achieve large scale injection operations requires MEMS or NEMS support cell that use anodic binding by printing techniques. The surfaces of holes are aligned on the cell support used to contain and fix individual cells for the injection. When well calibrated, the cellular support system enables a large number of cells to be injected using position control. The cell injection operation can be conducted in a sequence of Displacement - Injection - Displacement.

The injection is determined by the speed of injection, trajectory and forces applied to the cells (Fig. 3.). To improve the performance of the robot systems, it takes a capacitive cellular sensor based on multi-axial MEMS or NEMS to ensure the real-time reaction force of the micro-robotic system. The cellular MEMS or NEMS micro-sensor can be used in research to characterize the mechanical properties of the bio membrane.

The capacitive cellular force sensor based on double-axial MEMS is presented in Figure 4. is able to decompose the normal forces applied to a cell and tangential forces generated by improperly aligned cell samples. The micro-fabrication process can be developed to form a 3D structure with a high side aspect ratio using deep reactive ion etching (DRIE) - deep reactive ion etching on insulator silicon capsules (SOI). Thus, the constrained external skeleton and internal movable structure are connected by a four curved springs. A test load applied to the probe causes movement of the internal structure, changing the hole between each pair of interdigital capacitors. Therefore, full changing of the force applied decomposes the 
applied force. The interdigital capacitors are set orthogonally to allow the force sensor to break down the forces on both the $\mathrm{x}$ and $\mathrm{y}$ axis. The cellular force sensors used in experiments are able to decompose forces up to $25 \mu \mathrm{N}$ with a resolution of $0.01 \mu \mathrm{N}$.

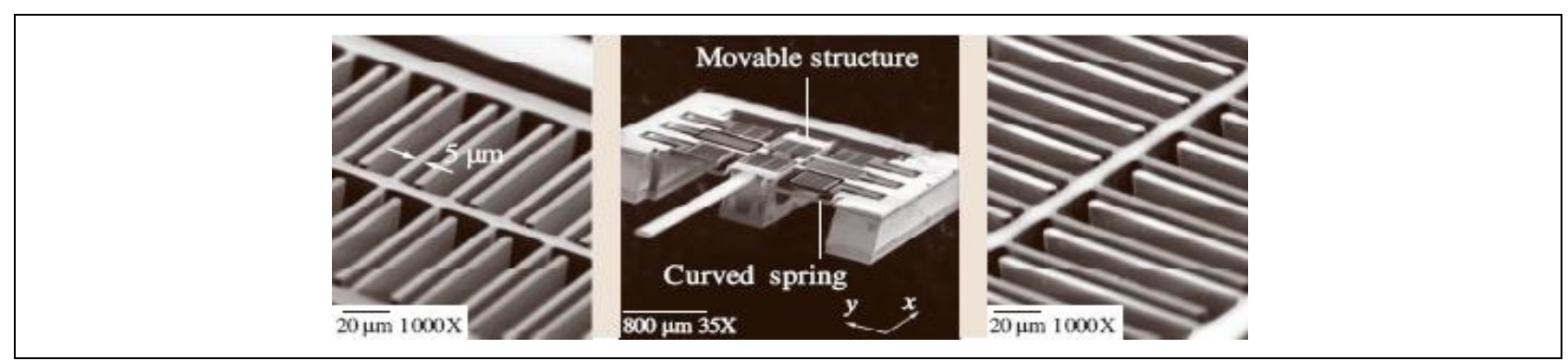

Fig. 4. The capacitive cellular force sensor based on double-axial MEMS

The tip structure affects the results of quantitative measurement of force. A standard injection pipette with a tip section with a diameter of $5 \mathrm{~mm}$ is attached to the micro-force sample cell sensors.

The robotic micro-system and cell force micro-sensor of great sensitivity are also applicable for studying the mechanical properties of the bio-membrane. The goal of the research is to obtain a general parametric model describing the behaviour of membrane deformation when an external load is applied. This parameterized model serves two purposes. The first goal is robotic bio-manipulation, which allows the recognition of the parameter so that the membrane deformation behaviour can be predicted. The second goal is the destruction of a thermodynamic model membrane where it is important to assess the mechanical behaviour of membranes. Thus the interpretation of the reported phenomena allows the cell mechanical strength volume during dehydration and its relationship to injury. Setting such a bio-membrane model will facilitate studies and results of cell damage.

Experiments and research show that micro-nano-robotics and MEMS and NEMS micro-nano-technologies can play important roles in biological studies of automatic bio-manipulation. Aided by micro-nano-robotics, the integration of power modules and force detectors in MEMS design, fabrication techniques and investigations is conducted to shape the mechanical properties of bio membranes, distort and manipulate cellular bio molecules and cells. These MEMS applications represents the endowments from MEMS \& NEMS Laboratory from INCDMTM Bucharest.

\section{Nanoelectromechanics systems NEMS}

The NEMS concept - nano-electro-mechanical systems - was addressed in the fundamental concept of nano-technology to ensure the precision required to process materials of up to $(0.05 \mathrm{~mm} \div 1 \mathrm{~nm})$, close to the distance between the atoms in a network of $0.3 \mathrm{~nm}$ that is the limit of resolution over the measurement length or confirmed lenght in measurable solid materials. Therefore, nanotechnology uses systems with intelligent technologies integrated for measurement, control and position in sub-micron processing. Therefore, nanotechnology must play an essential 
role in the manufacturing of highly precise components and assemblies ultrafine and also very precise and sometimes ultra precise NEMS.

NEMS treatment is very important and decisive for the design and implementation of future advanced technology, advanced intelligent platforms, industrial robots and robotic technology industrial services, technological devices and intelligent systems that measure and control system.

The fundamental purpose of NEMS is to integrate by all means, systems, technical and technological platforms in order to achieve intelligent mechatronic, electronic and informatic elements and components, with very high precision and accuracy and ultra-high-precise accuracy. NEMS provide micro-nano-technology processing, provide high accurate positioning, provid planning, decisions and system driving capability. To this end NEMS are integrated with the architecture of nanosensors, nano-transducers, nano-actuators, nano-computing units and special software and dedicated software for each application. These high secure NEMS provides all users with complex and higher, previously unsuspected functionality.

The integration of NEMS, also ensures the development of high-tech fields such as integronic robotics, a field that will be revolutionized, both for the industry and especially for the medical field as the intelligent measurement technique field will be developed in an unprecedented wat by nano-systems / intelligent testing, installation and integrated nano-measurement equipment, self-integrated in-process and postprocess in intelligent and in automated fabrication.

The NEMS technology applied today summarizes the main technological structural and applied field, highlighting the leading role played in the development of these areas, namely:

- technical / technological fields;

- system techniques;

- micro techniques;

- materials and effects;

- structural domains:

- integrable sensor architecture;

- integrable current architecture;

- signal processing components;

- application areas:

- process Engineering;

- medical and Biomedical Engineering;

- environmental technology and security;

- engineering robotics;

- appliance and office equipment technology;

NEMS development currently is in fact the development of products / systems of high intelligence and also contributes substantially to the systematization of nanotechnology. In this context, several key directions for the NEMS HIGH-TECH future development are highlighted: 
Gheorghe, G.; Voicu, A. C.; Ilie, I.; Gornoava, V. \& Anghel, C.: Intelligent Adaptr...

- the development of injtelligent nano-processing materials used for the manufacturing of elements and intelligent components, and particularly for integrated circuits with very high density;

- the development of circuits MOS integrated in processors in the field of microscopic or quantum interaction;

- the development of STMs and AFMs for systematized nanotechnology development;

- the development of nanoprocessing for advanced materials for optoelectronic components and nanoelectronics;

- the development of advanced nanotechnological components for nanomechanics;

- the development of devices for nano-mechatronic servopositioning nanotechnology;

- the development of ion beam processing of materials in nanotechnology;

- the development of laser processing of materials in nanotechnology;

- the development of computer simulation of atomic or molecular scale cutting;

- development of nano-technology and micro-machines;

The development and implementation of NEMS has been made possible by nanotechnology, due to new materials, intelligent materials and new materials and nanostructures through new andall innovated nanocomponents discovered so far.

On the other hand, the development and application of NEMS was made possible by research in intelligent engineering / micro-engineering / nano-engineering integrated with the new concepts and structures found in intelligent design and manufacturing (CAD / CAM), the new integrated scientific disciplines such as mechatronics / micronano-mechatronics, robotics / micro-nano-robotics and integronics / micro-nanointegronics, penutronics / micro-nano-pneutronics. NEMS structures partially or totally integrates elements / components needed to carry out their functions and subsystems and subsystems needed to carry out their functions and ensure the precision and accuracy in accordance with the needs of today.

As hardware structure, NEMS include:

- Nano-mechanical elements / components and subsystems;

- Nano-optical elements / components and subsystems;

- Nano-electronic elements / components and subsystems;

- nano-pneumatic elements / components and subsystems;

- nanoinformatice elements / components and subsystems;

As software structures, NEMS include:

- Programmes, special software;

- Programs dedicated software;

- Programs, software mix;

- Information processing;

- Information transfer;

- Self-diagnosis and information decision; 
- Information autocontrolling;

As an applicative destination, NEMS include:

- Technological applications in nano- processing with material removal;

- Technological applications in nano- processing without material removal;

- Technical applications of precise, very precise and ultra-precise positioning and orientation;

- Technical applications of precise, very precise and ultra-precise measuring, testing, adjusting and control;

- Technical / technological applications in industry;

- Technical / technological in medicine;

- Technical / technological in computerization of industry, economy and society;

NEMS development continues in the near future by modernizing and developing operation, construction, computerization and artificial intelligence based on the development of nano-sciences, nano-technologies and nano-materials. In this respect, the achievements of nano-sciences integrate with NEMS and their principles, with the new concepts and new philosophies applied to the future NEMS and last but not least, with the new discoveries in science, technique and technology. Also, the field of nano-technologies integrates with the intelligent and highly advanced nanoequipments and especially with the new high-tech concept of "rapid prototypying". The field od nanoaterials integrates with intelligent and hiper-structural materials and new scientific discoveries related to atomic and subatomic bond matrices for which new nano-processing technologies are saught, at atom and even and even subatomic scale. In multi-purpose structure, NEMS, in a new generation of development, will use new intelligent materials and / or nano-materials based on new technologies of building clusters of atoms of the same material, will use new nano-fabrication and nano-assembly nano-technologies and will meet new generations of development, at nano-scale.

\subsection{NEMS Applications}

NEMS applications have began to be multiplied to more and more in industries and especially in high-tech industrial fields, to ensure precision, accuracy and maintenance processes and measuring, regulating and control processes.Especially, the types of NEMS are available in a variety of intelligent integration solutions and applications for the micro and nano level. Further, the next generation of NEMS dedicated to robotics and micro-nano-robotics will be presented. Some of the robotic and micro-nano-robotic systems / micro- systems outlined, are:

(a) Cartesian Nano-manipulator (x, y, z) with nano resolution (Fig. 5.): 


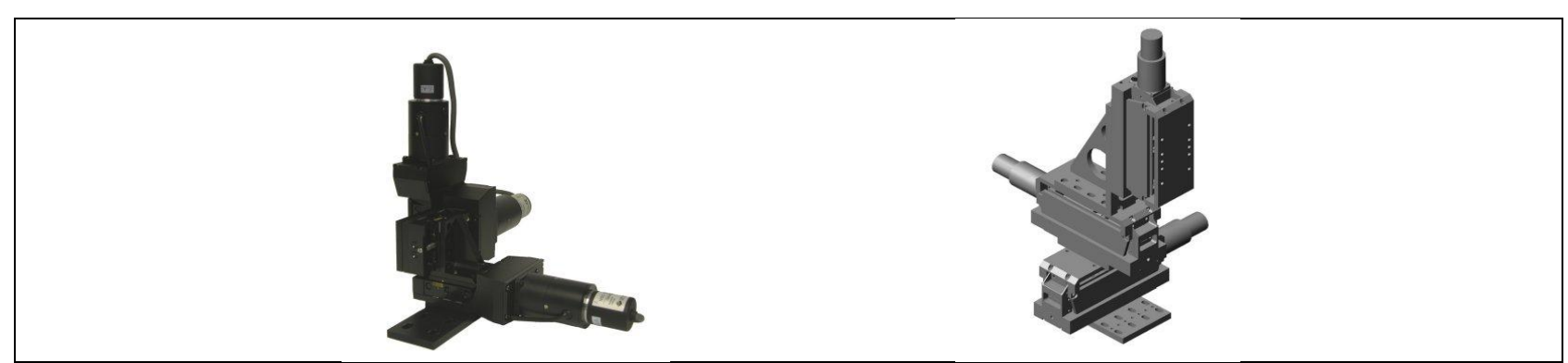

Fig. 5. Cartesian Nano-manipulator $(\mathrm{x}, \mathrm{y}, \mathrm{z})$ with nano resolution

- displacement increment: $0.4 \mathrm{~nm}$;

- working range: $10 \div 100 \mathrm{~mm}$;

- working speed: $2000 \mathrm{~m} / \mathrm{s}$;

- displacement: continuous or in steps;

- acceleration time at maximum speed: $0.3 \mathrm{~ms}$;

- response time: $10 \mathrm{~ms}$;

- nano-positioning and nano-movement: ideal;

- controller: high precision multi-channel

The Cartesian Nano-manipulator with nano-resolution comprises modular / standard units, such as the short linear motion transfer mode with ultra-high stability and the long linear motion transfer mode with working range of $100 \mathrm{~mm}$ (Fig. 6.).
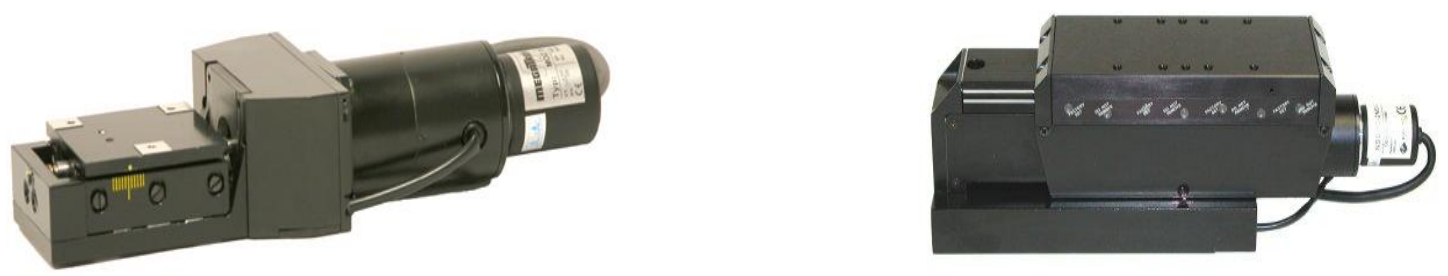

Fig. 6. The Cartesian Nano-manipulator with nano-resolution

(b) Micro-robotic system with 6 axes for comolex alignment with high micropositioning accuracy (Fig. 7.):

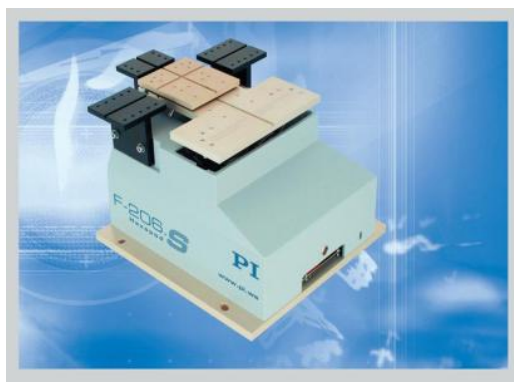

Fig. 7. Micro-robotic system with 6 axes for comolex alignment with high micropositioning accuracy

- parallel kinematic structure with 6 orientations;

- actuators with resolution of $0.033 \mathrm{~nm}$; 
- repeatability in space: $0.3 \mathrm{~nm}$;

- compact serial kinematic system and better dynamics;

- precise scanning and alignment;

- highlights vizualization and Cartesian coordinates control;

- digital controller with LabView program;

- integrated alignment routines;

(c) The "ProScan" for automating microscopes (Fig. 8.):
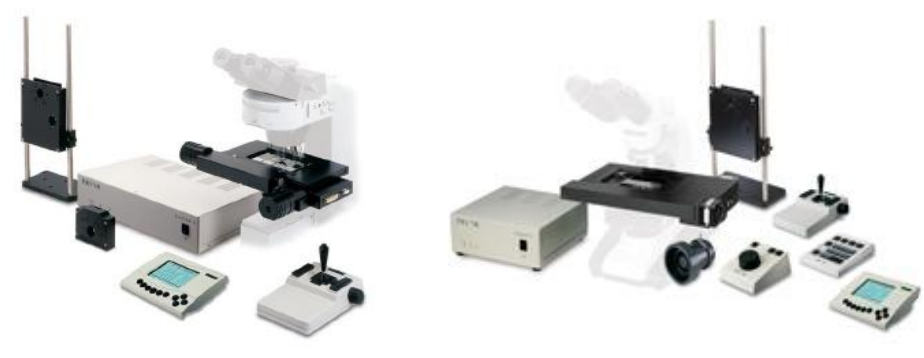

Fig. 8. The "ProScan" for automating microscopes

Fig. 9. "OptiScan» Automation microscopes

- controller: high speed; travel: motorized; precision: very high;

- buildings: modular; use: automatic micro-scopes; communication: Serial TTL inputs and outputs; routine: autofocusare; software: a higher level;

(d) "OptiScan» Automation microscopes (Fig. 9.):

- controller: High Speed; travel: motorized; mechanism: by focusing;

- software: image analysis; further development: upgrades;

- applications: data acquisition, scanning, metallurgical analysis, etc..;

- development: upgrades, data transfer rate - $32 \mathrm{~kb} / \mathrm{s}$, high speed positioning, integrated sensor positioning, generating high forces, vertical and horizontal operations;

(e) Microrobot positioning "hexapod 6-axes, with parallel kinematics" (Fig. 10.):

- controller; orientation: plane and space;

- capacity (vertical): $200 \mathrm{~kg}$; resolution: ultra-high;

- repeatability: $\pm 1 \mathrm{~mm}$; sensor resolution: $0.005 \mathrm{~mm}$;

- multi-axes linear and rotational scanning; pivot points: virtual;

- algorithm: sophisticated for the cotroller; MTBF: 20,000 h:

These NEMS applications represents the endowments from MEMS \& NEMS Laboratory from INCDMTM Bucharest. 
Gheorghe, G.; Voicu, A. C.; Ilie, I.; Gornoava, V. \& Anghel, C.: Intelligent Adaptr...

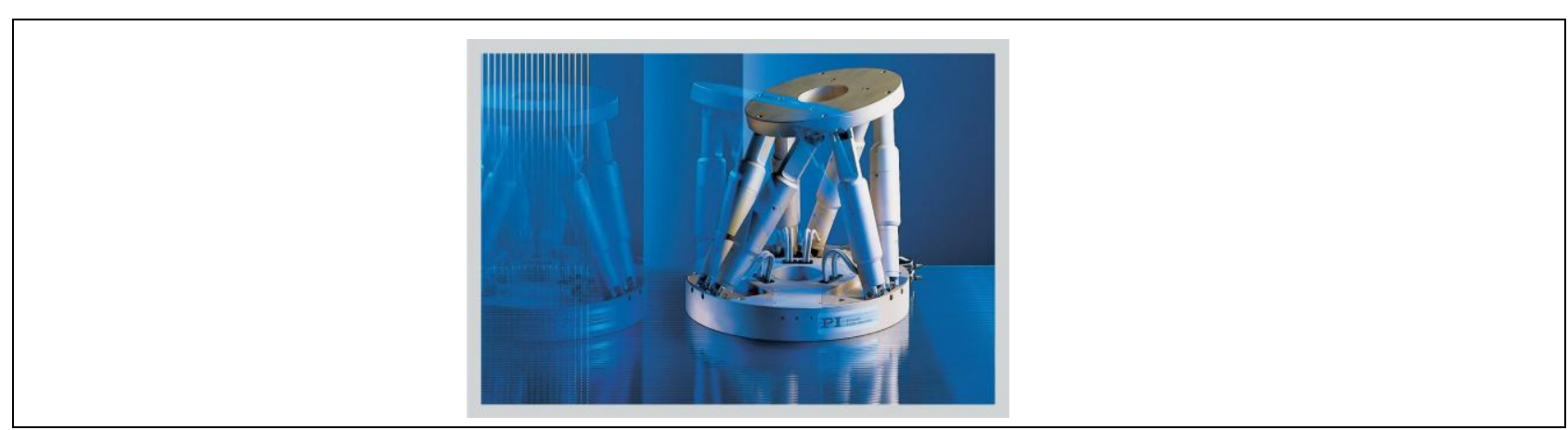

Fig. 10. Microrobot positioning "hexapod 6-axes, with parallel kinematics"

\section{Conclusions}

Various models built based on the open literature show significant differences. The deflection and the resonant frequency of two micro-cantilever beams are experimentally measured and compared to the numerical methods for static and dynamic models. Different models fit different experimental data.

The findings on this work bring more understanding on the phenomena at microsize level. Nonlinear analysis is important in MEMS accurate modelling. The nonlinear theories are difficult to apprehend and they represent a step away from the reasoning of engineering which aims simplified theories. However, when linearizing differential equations in micro-systems modelling, that operation should be carried with caution. For this reason, it seems reasonable that for all MEMS and NEMS applications one should perform analysis solving the nonlinear differential equations. The problem of this scientific paper was the building of various models of intelligent adaptronics MEMS and NEMS, based on calculations regarding the influence of geometry nonlinearity and material nonlinearity, who was used. The building of intelligent adaptronics MEMS and NEMS, based on a study on realised MEMS and NEMS models at national and international level in the existing literature from this domain. INCDMTM realised theoretical and practical researches in the MEMS and NEMS Laboratory in order to achieve his own MEMS and NEMS models, characterised by micro technical integrative solutions for intelligent applications.

\section{References}

Gheorghe, I.G., Carstoiu, A., Despa V. (2011). Intelligent integrative Micro-Nano Robotics. Proceeding of 22nd DAAAM Symposium, Viena, Austria

Reddy, J.N. (2004). An introduction to nonlinear finite element analysis, Oxford University Press, USA

Belendez, T. (2002). Large and small deflection of a cantilever beam. European Journal of Physics, Vol., No.23,) page numbers (371-375)

Gheorghe, I.G., Liliana Laura Badita (2013). Micro-nanometrologically and topographic characterization of nanostructured surfaces. Procedia Engineering Proceedings of $24^{\text {th }}$ DAAAM International Symposium, Zadar, Croatia

Gheorghe, I.G. (2010). Intelligent instrumentation engineering, Cefin Publishing House, ISBN: 978-606-8261-00-3 